\title{
MEASURE ALGEBRAS ON ABELIAN GROUPS
}

\section{WALTER RUDIN ${ }^{1}$}

The recent developments in the general field of Fourier analysis which I wish to describe, illustrate the algebraic point of view which has established itself here as well as in most other parts of analysis, and which has been most fruitful in suggesting new and interesting problems. Typically, a question will pose itself in algebraic terms, will then be transformed into a problem which can be attacked by the methods of classical analysis, and the algebraic aspect will reappear in the solution.

To cite just one example now, one may ask for the set of all automorphisms of the group algebra of the circle group; this turns out to be equivalent to the problem of finding all permutations of the set of all integers which carry Fourier series into Fourier series; the solution of this analytical problem involves arithmetic progressions, i.e., cosets of the subgroups of the additive group of the integers, and the group theoretic nature of the problem becomes apparent (the result is described in 3.3(d) below).

This is a large subject, and I will restrict myself to those topics with which I have had close personal contact. This choice will, of course, result in the omission of much that is interesting. In particular, I will say nothing about what is perhaps the most intriguing of the open problems in Fourier analysis: the problem of spectral synthesis, or, equivalently, the problem of finding all closed ideals in $L^{1}(G)$.

I. Preliminaries. 1.1. Unless the contrary is explicitly stated, any group mentioned in the sequel will be abelian and locally compact, with addition as group operation. Associated with every such group $G$ there is a measure $m$ (not identically 0 or $\infty$ ), the so-called Haar measure of $G$, with the following properties $[6 ; 7 ; 21 ; 31]: m$ is a non-negative completely additive set function defined for all Borel sets in $G$, which is regular (i.e., $m(E)=\sup m(K)=\inf m(V)$, where $K$ ranges over all compact subsets of $E$ and $V$ ranges over all open supersets of $E$ ), and which is translation invariant:

An address delivered before the Cambridge meeting of the Society on August 28, 1958 by invitation of the Committee to Select Hour Speakers for Summer Meetings; received by the editors November 19, 1958.

${ }^{1}$ Research Fellow of the Alfred P. Sloan Foundation. 
These conditions characterize $m$, up to a positive multiplicative constant. When $G$ is compact, it is customary to normalize $m$ so that $m(G)=1$; when $G$ is discrete, any set consisting of a single point of $G$ is assigned the measure 1. In any case, $m(K)$ is finite if $K$ is compact, $m(V)$ is positive if $V$ is open, and $m(G)$ is finite if and only if $G$ is compact.

1.2. Definition of $M(G)$. For any of the groups $G$ under consideration, $M(G)$ is the set of all complex-valued completely additive Borel measures on $G$ which are regular in the following sense: any measure $\mu$ with the above properties has a decomposition $\mu=\mu_{1}-\mu_{2}$ $+i \mu_{3}-i \mu_{4}$, with $\mu_{j} \geqq 0$; it is required that each $\mu_{j}$ be regular in the sense in which this term was defined in 1.1. The requirement that $\mu(E)$ be a complex number is meant to exclude the possibility that $\mu(E)=\infty$. Thus the Haar measure of $G$ belongs to $M(G)$ if and only if $G$ is compact.

A norm is introduced in $M(G)$ by defining

$$
\|\mu\|=\sup \sum\left|\mu\left(E_{i}\right)\right| \quad(\mu \in M(G)),
$$

the sup being taken over all finite collections of pairwise disjoint Borel sets $E_{i}$ whose union is $G$; it is easy to see, via the Hahn decomposition theorem [7, p. 121], that $\|\mu\|<\infty$ for every $\mu \in M(G)$.

We say that $\mu$ is concentrated on $E$ if $\mu(A)=0$ whenever $A$ and $E$ are disjoint. If $\mu$ is concentrated on a countable set, then $\mu$ is discrete; if $\mu(E)=0$ for every countable set $E$, then $\mu$ is continuous; if $\mu(E)=0$ whenever $m(E)=0$, where $m$ is the Haar measure of $G$, then $\mu$ is absolutely continuous; finally, if $\mu$ is concentrated on a set $E$ with $m(E)=0$, then $\mu$ is singular.

1.3. Definition of $L^{1}(G)$. The set of all complex-valued functions on $G$ which are integrable with respect to the Haar measure of $G$ is denoted by $L^{1}(G)$. Every $f \in L^{1}(G)$ has associated with it an absolutely continuous measure $\mu_{f} \in M(G)$ :

$$
\mu_{f}(E)=\int_{E} f d m .
$$

Conversely, the Radon-Nikodym theorem [7, p. 128] shows that every absolutely continuous $\mu \in M(G)$ is $\mu_{f}$ for some $f \in L^{1}(G)$. If, as is customary, we identify any two functions in $L^{1}(G)$ whose values differ only on a set of Haar measure zero, then the correspondence between $f$ and $\mu_{f}$ is one-to-one, and we may therefore regard $L^{1}(G)$ as a subset of $M(G)$. It is convenient to use $f$ and $\mu_{f}$ interchangeably. 
The restriction of the norm defined by (1.2.1) to $L^{1}(G)$ coincides with the usual norm in spaces of integrable functions:

$$
\|f\|=\left\|\mu_{f}\right\|=\int_{G}|f| d m \quad\left(f \in L^{1}(G)\right) .
$$

Note that $L^{1}(G)=M(G)$ if and only if $G$ is discrete.

1.4. Algebraic Operations in $M(G)$. It is obvious how $M(G)$ can be made into a linear space: if $\mu, \lambda \in M(G)$, define

$$
\begin{aligned}
(\mu+\lambda)(E) & =\mu(E)+\lambda(E), \\
(c \mu)(E) & =c \cdot \mu(E),
\end{aligned}
$$

for every Borel set $E$ in $G$ and for every complex number $c$. With these operations, and with the norm (1.2.1), $M(G)$ is in fact a Banach space, and $L^{1}(G)$ is a closed subspace of $M(G)$.

Multiplication of two measures is defined by convolution: If $\mu, \lambda \in M(G)$, let $\mu \times \lambda$ denote their product measure [7] in the Cartesian product space $G^{2}$, and associate with each Borel set $E$ in $G$ the set

$$
E_{(2)}=\left\{(x, y) \in G^{2} \mid x+y \in E\right\} .
$$

Put

$$
(\mu * \lambda)(E)=(\mu \times \lambda)\left(E_{(2)}\right),
$$

and call the set function $\mu * \lambda$ so defined the convolution of $\mu$ and $\lambda$.

It is not hard to verify that $\mu * \lambda \in M(G)$, that $\mu * \lambda=\lambda * \mu$ (since $G$ is abelian), that convolution is associative, and that

$$
\|\mu * \lambda\| \leqq\|\mu\| \cdot\|\lambda\| ;
$$

each of these verifications is an application of Fubini's theorem.

Thus, with convolution as multiplication, $M(G)$ is a Banach algebra. There is a unit element in $M(G)$, namely the measure $u$ such that $u(E)=1$ if $0 \in E$ and $u(E)=0$ otherwise; $u$ is the unit mass concentrated at 0 , and $\mu * u=\mu$ for every $\mu \in M(G)$.

The convolution of more than two measures can be defined by associativity; another (equivalent) definition, well adapted to some applications (see 2.3) is as follows: if $\mu_{1}, \cdots, \mu_{n} \in M(G)$, associate with each $E \subset G$ the set

$$
E_{(n)}=\left\{\left(x_{1}, \cdots, x_{n}\right) \in G^{n} \mid x_{1}+\cdots+x_{n} \in E\right\},
$$

and put

$$
\left(\mu_{1} * \mu_{2} * \cdots * \mu_{n}\right)(E)=\left(\mu_{1} \times \mu_{2} \times \cdots \times \mu_{n}\right)\left(E_{(n)}\right),
$$


where the measure on the right is the ordinary product measure.

We also define

$$
\mu^{1}=\mu, \quad \mu^{n}=\mu * \mu^{n-1}, \quad \mu^{0}=u .
$$

1.5. Group Algebras. By the Fubini theorem, (1.4.3) can be rewritten, for every Borel set $E$, in the form

$$
(\mu * \lambda)(E)=\int_{G} \mu(E-x) d \lambda(x) .
$$

From this one immediately concludes the following: if $\mu$ is absolutely continuous, so is $\mu * \lambda$; if $\mu$ is continuous, so is $\mu * \lambda$; if both $\mu$ and $\lambda$ are discrete, so is $\mu * \lambda$. In other words, $L^{1}(G)$ is a closed ideal in $M(G)$, and so is the set of all continuous $\mu \in M(G)$; the discrete measures form a closed subalgebra of $M(G)$.

The algebra $L^{1}(G)$ is usually called the group algebra of $G$, although this term should perhaps have been reserved for $M(G)$. In the classical cases ( $G$ finite) $L^{1}(G)$ and $M(G)$ coincide, and it is a matter of taste whether one wishes to consider the elements of a finite-dimensional group algebra as functions on $G$ or as measures on $G$. However, $M(G)$ does have one advantage: its definition does not depend on the construction of Haar measure.

1.6. In this connection it may be of interest to note that the absolutely continuous measures in $M(G)$ can be characterized without any reference to Haar measure:

Theorem. A measure $\mu$ in $M(G)$ is absolutely continuous if and only if $\mu(E-x)$ is a continuous function of $x$, for every Borel set $E$ in $G$.

Proof. Suppose $\mu=\mu_{f}$ for some $f \in L^{1}(G)$, and define $f_{x}$, for $x \in G$, by $f_{x}(y)=f(y-x)$. Then $\left\|f_{x}-f\right\| \rightarrow 0$ as $x \rightarrow 0[21$, p. 118], and the continuity of $\mu(E-x)$ follows from the relation $\mu_{f}(E-x)=\mu_{f_{x}}(E)$.

To prove the converse, suppose $m(E)=0$, and let $g$ be the characteristic function of a Borel set $A$ such that $m(A)<\infty$. Then

$$
\int_{A} \mu(E-x) d m(x)=(g * \mu)(E) .
$$

Since $g \in L^{1}(G)$ and since $L^{1}(G)$ is an ideal in $M(G)$, the right member of (1.6.1) is 0 . Hence $\mu(E-x)=0$ for almost all $x$; by continuity, $\mu(E)=0$, and the theorem is proved.

1.7. Characters, DUAL Groups, Fourier transforms, AND FOURIER-STIELTJES TRANSFORMS. A continuous character on a group $G$ is a continuous complex function $\gamma$ on $G$, whose values will be denoted by $(x, \gamma)$, such that $|(x, \gamma)|=1$ and 


$$
(x+y, \gamma)=(x, \gamma) \cdot(y, \gamma)
$$

$(x, y \in G)$.

The set of all such $\gamma$ forms a group $\Gamma$, the dual group of $G$, if addition is defined by

$$
\left(x, \gamma_{1}+\gamma_{2}\right)=\left(x, \gamma_{1}\right) \cdot\left(x, \gamma_{2}\right) \quad\left(x \in G ; \gamma_{1}, \gamma_{2} \in \Gamma\right) ;
$$

$\Gamma$ is usually given the compact-open topology: to every compact set $K$ in $G$ and to every $\epsilon>0$ there corresponds a neighborhood of 0 which consists of all $\gamma$ such that

$$
|1-(x, \gamma)|<\epsilon \quad(x \in K) .
$$

With this topology, $\Gamma$ is a locally compact abelian group, and $G$ is in turn the dual group of $\Gamma$ (this is the celebrated Pontryagin duality theorem [3]). If $G$ is compact then $\Gamma$ is discrete, and vice versa.

With every $f \in L^{1}(G)$ is associated its Fourier transform $\hat{f}$ :

$$
\hat{f}(\gamma)=\int_{G}(-x, \gamma) f(x) d m(x)
$$

if $\mu \in M(G)$, its Fourier-Stieltjes transform (abbreviated to F. S. transform in the sequel) is the function $\hat{\mu}$ defined analogously by

$$
\hat{\mu}(\gamma)=\int_{G}(-x, \gamma) d \mu(x)
$$

It is clear that (1.7.4) and (1.7.5) coincide for absolutely continuous $\mu$ : in the notation of $1.3, \hat{f}=\hat{\mu}_{f}$.

A concise presentation of the basic theorems about these transforms may be found in [3]. The functions $\hat{\mu}$ are uniformly continuous and bounded on $\Gamma$, the correspondence between $\mu$ and $\hat{\mu}$ is one-to-one, and the mapping $\mu \rightarrow \hat{\mu}$ is thus an isomorphism of the algebra $M(G)$ onto an algebra of continuous functions on $\Gamma$ : the mapping is clearly linear, and if $\sigma=\mu * \lambda$, then

$$
\hat{\sigma}(\gamma)=\hat{\mu}(\gamma) \hat{\lambda}(\gamma)
$$

$$
(\gamma \in \Gamma) .
$$

It follows that the mapping $\mu \rightarrow \hat{\mu}(\gamma)$, for any fixed $\gamma \in \Gamma$, is a complex homomorphism of $M(G)$, and the fact that $\hat{\mu}$ determines $\mu$ implies that $M(G)$ is a semi-simple Banach algebra [21, p. 76].

1.8. The Gelfand Transforms of Measures. Being a commutative Banach algebra with unit, $M(G)$ has a compact maximal ideal space $\Delta$. We may consider $\Delta$ to be the set of all homomorphisms $h$ of $M(G)$ onto the complex field; the topology of $\Delta$ is the weakest one in which the functions $\hat{\mu}$ defined on $\Delta$ by

$$
\hat{\mu}(h)=h(\mu)
$$$$
(\mu \in M(G), h \in \Delta)
$$ 
are continuous. We call $\hat{\mu}$ the Gelfand transform of $\mu$.

We saw in 1.7 that every $\gamma \in \Gamma$ defines an element $h_{\gamma}$ of $\Delta$, by the formula

$$
h_{\gamma}(\mu)=\hat{\mu}(\gamma)=\int_{G}(-x, \gamma) d \mu(x) \quad(\mu \in M(G)) .
$$

We identify $\gamma$ and $h_{\gamma}$, and thus embed $\Gamma$ in $\Delta$; this embedding is a homeomorphism of $\Gamma$ into $\Delta$, and the Fourier-Stieltjes transforms on $\Gamma$ are precisely the restrictions of the Gelfand transforms to $\Gamma$ (so that the two ways in which the notation $\hat{\mu}$ was introduced are compatible).

1.9. The Circle Group and the Integers. To cite just one familiar example, let $G$ be the circle group $C$, i.e., the set of all complex numbers of absolute value 1 , with multiplication as group operation (or, equivalently, the set of all real numbers, with addition $\bmod 2 \pi$ ). The dual group of $C$ is $J$, the additive group of all integers; the characters are of the form

$$
\left(e^{i \theta}, n\right)=e^{i n \theta}
$$$$
\left(e^{i \theta} \in C, n \in J\right),
$$

and the F. S. transform $\hat{\mu}$ of any $\mu \in M(C)$ is given by

$$
\hat{\mu}(n)=\int_{0}^{2 \pi} e^{-i n \theta} d \mu(\theta)
$$

the numbers $\hat{\mu}(n)$ are usually called the F. S. coefficients of $\mu$. The Haar measure of $C$ is the ordinary Lebesgue measure, divided by $2 \pi$. then

$$
\hat{\mu}\left(e^{i \theta}\right)=\sum_{n=-\infty}^{\infty} \mu(\{n\}) e^{-i n \theta} .
$$

In other words, the F. S. transforms on $C$ are precisely those functions which are sums of absolutely convergent trigonometric series.

II. The maximal ideal space of $M(G) .2 .1$. We saw in 1.8 that $\Gamma$ is a subset of $\Delta$, the maximal ideal space of $M(G)$. In one important case, $\Gamma=\Delta$ : this happens when $G$ is discrete, for then $M(G)=L^{1}(G)$, and it is known $[21$, p. 136] that $\Gamma$ is always the maximal ideal space of $L^{1}(G)$. If $G$ is not discrete, however, then $\Gamma$ is not compact, and hence $\Gamma$ is a proper subset of $\Delta$. The question arises whether one can say anything more specific about the relation between $\Gamma$ and $\Delta$.

2.2. In 1950, Sreider [30] discovered a surprising fact: if $G$ is the additive group of the real line, then $\Gamma$ is not dense in $\Delta$, and $M(G)$ is not symmetric (i.e., the set of all Gelfand transforms is not closed 
under complex conjugation). This was all the more surprising since the F. S. transforms are always closed under conjugation: if $\sigma(E)$ $=\bar{\mu}(-E)$, then $\hat{\sigma}(\gamma)$ is the complex conjugate of $\hat{\mu}(\gamma)$, for all $\gamma \in \Gamma$.

Hewitt [13] extended this result to groups $G$ in which every neighborhood of 0 contains an element of infinite order; the essential part of his proof was simplified in [25]. Recently Williamson [37] has succeeded in proving the same thing for every nondiscrete $G$. I will sketch a proof of this interesting result, using the method of [25] to prove the lemma:

2.3. LEMMA. On every nondiscrete locally compact abelian group $G$ there is a non-negative continuous measure $\mu \in M(G)$, such that $\mu(E)$ $=\mu(-E),\|\mu\|=1$, and

$$
\left\|\sum_{i=0}^{n} a_{i} \mu^{i}\right\|=\sum_{i=0}^{n}\left|a_{i}\right|
$$

for every choice of complex numbers $a_{0}, \cdots, a_{n}$.

(The measures $\mu^{i}$ are defined by (1.4.7).)

Proof. One of two cases must occur [25]: either every neighborhood of 0 in $G$ contains an element of infinite order, or $G$ contains a compact open subgroup of bounded order. In the former case, put $W(G)=\infty$. In the latter case, there is a compact open group $K$ in $G$ and there is an integer $W(G) \geqq 2$, such that (i) the order of no element of $K$ exceeds $W(G)$, (ii) every neighborhood of 0 in $G$ contains an element of order $W(G)$. With this notation, the following proposition holds:

There exists a set $Q$ in $G$, homeomorphic to the Cantor set, with the following property: if $g_{1}, \cdots, g_{p}$ are distinct points of $Q$, if $n_{1}, \cdots, n_{p}$ are integers, not all 0 , such that $\left|n_{i}\right|<W(G)$, then

$$
n_{1} g_{1}+\cdots+n_{p} g_{p} \neq 0 \text {. }
$$

For $W(G)=\infty$, this was proved in [25]. In the other cases the proof is quite analogous; $Q$ is constructed as a subset of $K$, by a suitable imitation of the usual construction of a Cantor set on the line as the intersection of sets $E_{n}$ which are unions of $2^{n}$ intervals.

Having $Q$, we put $P=Q \cup(-Q)$, and take for $\mu$ any continuous non-negative measure which is concentrated on $P$, such that $\|\mu\|=1$ and $\mu(E)=\mu(-E)$; since $P=-P$, this can be done. Since $\mu^{n}$ is concentrated on $P_{n}$, where $P_{1}=P$ and $P_{n+1}=P_{n}+P$, it is enough to show that $m<n$ implies

$$
\mu^{n}\left(P_{m}\right)=0 .
$$


Fix $m<n$, and let $S$ be the set of all $\left(x_{1}, \cdots, x_{n}\right) \in G^{n}$ such that

$$
x_{1} \in P, \cdots, x_{n} \in P ; \quad x_{1}+\cdots+x_{n} \in P_{m} .
$$

Define $\mu_{(n)}$ by: $\mu_{(1)}=\mu, \mu_{(n)}=\mu \times \mu_{(n-1)}$, so that $\mu_{(n)}$ is a measure on $G^{n}$. Since $\mu$ is concentrated on $P,(1.4 .6)$ shows that

$$
\mu^{n}\left(P_{m}^{\cdot}\right)=\mu_{(n)}(S) .
$$

Suppose $\left(x_{1}, \cdots, x_{n}\right) \in S$. By (2.3.4) there are points $y_{1}, \cdots, y_{m}$ $\in P$, such that

$$
x_{1}+\cdots+x_{n}=y_{1}+\cdots+y_{m} .
$$

The definition of $P$ shows that $x_{i}=\epsilon_{i} g_{i}$, with $\epsilon_{i}= \pm 1, g_{i} \in Q$. If $g_{i} \neq g_{j}$ whenever $i \neq j$, the fact that $m<n$ leads to a relation which contradicts (2.3.2). Hence $x_{i} \pm x_{j}=0$ for some $i \neq j$. Since $\mu$ is continuous, the set of all points in $G^{(n)}$ at which any of the relations $x_{i} \pm x_{j}=0$ holds, has measure 0 with respect to $\mu_{(n)}$. It follows that $\mu_{(n)}(S)=0$, and the lemma is proved.

2.4. ThEOREM [37]. Suppose $G$ is a locally compact, abelian, nondiscrete group. Then

(a) $M(G)$ is not symmetric;

(b) the closure of $\Gamma$ in $\Delta$ does not contain the Silov boundary of $\Delta$;

(c) the Wiener-Pitt phenomenon occurs.

For the definition of Silov boundary we refer to [21, p. 80]. By (c) we mean that there exists a F.S. transform on $\Gamma$ whose reciprocal is bounded but is not a F. S. transform; on the real line such an example was constructed in [36]. We note that each of the three assertions of the theorem implies that $\Gamma$ is not dense in $\Delta$.

Proof. Take $\mu$ as in Lemma 2.3, and put $\sigma=u-\mu^{2}$, where $u$ is the unit of $M(G)$. By (2.3.1),

$$
\left\|\sigma^{n}\right\|=\left\|\sum_{0}^{n}\left(\begin{array}{l}
n \\
k
\end{array}\right)(-1)^{k} \mu^{2 k}\right\|=\sum_{0}^{n}\left(\begin{array}{l}
n \\
k
\end{array}\right)=2^{n} \quad(n=1,2,3, \cdots),
$$

so that the spectral norm of $\sigma, \lim \left\|\sigma^{n}\right\|^{1 / n}$, is $2[21, \mathrm{p} .75]$. Thus there is an $h \in \Delta$ such that $|h(\sigma)|=2$. Since $\|\mu\|=1$, we have $\left|h\left(\mu^{2}\right)\right| \leqq 1$, and the equation $\left|1-h\left(\mu^{2}\right)\right|=2$ implies that $h\left(\mu^{2}\right)=-1$ and $h(\sigma)=2$.

Since $\mu(-E)=\mu(E)$ and $\mu$ is real, $\hat{\mu}(\gamma)$ is real on $\Gamma$. However, $\hat{\mu}(h)=h(\mu)= \pm i$. If the complex conjugate of $\hat{\mu}$ were the Gelfand transform of some $\nu \in M(G)$, then $\hat{\mu}-\hat{\nu}$ would vanish at every point of $\Gamma$, so that $\mu=\nu$. But $\hat{\mu}(h) \neq \hat{\nu}(h)$. This contradiction proves $(a)$.

Since $0 \leqq \hat{\mu}^{2}(\gamma) \leqq 1$ on $\Gamma$, and $\hat{\sigma}=1-\hat{\mu}^{2}$, we have $0 \leqq \hat{\sigma}(\gamma) \leqq 1$ on $\Gamma$; since $\hat{\sigma}(h)=2,|\hat{\sigma}|$ does not attain its maximum over $\Delta$ at any point of the closure of $\Gamma$, and (b) follows. 
To prove (c), put $\tau=u+\mu^{2}$. Then $1 \leqq \hat{\tau}(\gamma) \leqq 2$ on $\Gamma$, but $\tau(h)$ $=1+h\left(\mu^{2}\right)=0$, so that $\tau^{-1}$ does not exist in $M(G)$.

This completes Theorem 2.4 .

2.5. There is not much known about the topological properties of $\Delta$ (Sreider's description of the points of $\Delta$ by means of "generalized characters" [30] seems to throw little light on the problem), but here are a few observations (it is assumed throughout that $G$ is not discrete).

(a) The Gelfand transforms of the absolutely continuous measures in $M(G)$ vanish outside $\Gamma$.

If $h$ does not annihilate every $f \in L^{1}(G)$, then there is a $\gamma \in \Gamma$ such that $h(f)=\hat{f}(\gamma)$ for all $f \in L^{1}(G)$, and $\hat{f}_{0}(\gamma)=1$ for some $f_{0} \in L^{1}(G)$. For any $\mu \in M(G)$, the measure $\sigma=\mu * f_{0}$ is absolutely continuous, and so $h(\mu)=h(\mu) h\left(f_{0}\right)=h(\sigma)=\hat{\sigma}(\gamma)=\hat{\mu}(\gamma)$. In other words, $h \in \Gamma$.

(b) Corollary. $\Gamma$ is an open subset of $\Delta$.

(c) Although $\Gamma$ is not dense in $\Delta, \Delta$ is "closely attached" to $\Gamma$ in the following sense:

$\Delta$ is not the union of two nonempty open-closed sets, one of which contains $\Gamma$.

If it were, a well-known theorem of Silov [29] would imply the existence of some $\mu \in M(G)$ such that $\hat{\mu}(\gamma)=0$ on $\Gamma$, but $\hat{\mu}(h)=1$ on some nonempty subset of $\Delta-\Gamma$, which is impossible.

(d) Corollary. If $\Gamma$ is connected, so is $\Delta$.

(e) There are singular measures in $M(C)$ whose Gelfand transforms vanish outside $J$. I.e., the converse of (a) is not true. (See 1.9 for notation.)

Indeed, Salem [28, Theorem III] has shown that there are singular measures $\mu \in M(C)$ whose F. S. coefficients are $O\left(|n|^{-\alpha}\right)$ for some $\alpha>0$; hence $\mu^{k} \in L^{2}$ for some positive integer $k$, and (a) implies that $[h(\mu)]^{k}=h\left(\mu^{k}\right)=0$ outside $J$.

(f) There is a singular measure $\mu \in M(C)$ such that $\hat{\mu}(n) \rightarrow 0$ as $|n| \rightarrow \infty$, but such that $\hat{\mu}(h) \neq 0$ for some $h \in \Delta-J$. (Here $\Delta$ is of course the maximal ideal space of $M(C)$.)

It is known that there is a perfect set $P$ on $C$ which is not a basis and which carries a positive continuous measure $\mu$ whose F. S. coefficients tend to 0 as $|n| \rightarrow \infty \quad[30$, p. $25 ; 28$, Theorem IV]. Let $F$ be the regular family of sets (in the sense of Raikov $[30, \mathrm{p} .15 ; 5, \mathrm{pp}$. 184-187]) generated by $P$, let $\sigma_{F}$ be the projection of $\sigma$ on $F$, and define $h(\sigma)=\sigma_{F}(C)$. Then $h \notin J$, but $h(\mu)>0$.

2.6. The following problems might be of interest:

(a) What is the Silov boundary of $\Delta$ ? Is it all of $\Delta$ ? 
(b) Is there any subset $S$ of $\Delta$, larger than the closure of $\Gamma$, on which the restrictions of the Gelfand transforms are closed under conjugation?

(c) Let $R(G)$ be the algebra of all self-adjoint elements of $M(G)$. That is, $\mu \in R(G)$ if and only if there is a $\sigma \in M(G)$ whose Gelfand transform is the complex conjugate of the Gelfand transform of $\mu$. $R(G)$ contains all $\mu$ such that $\hat{\mu}=0$ outside $\Gamma$ (or outside any set $S$ with the properties $(b)) ; R(G)$ also contains all discrete measures on $G$. Is there any intrinsic description of those measures which belong to $R(G)$ ?

III. Isomorphisms of group algebras. 3.1. Consider two locally compact abelian groups, $G_{1}$ and $G_{2}$, with dual groups $\Gamma_{1}$ and $\Gamma_{2}$, and suppose that $\tau$ is an isomorphism of $G_{2}$ onto $G_{1}$ (an isomorphism of two topological groups is of course also required to be a homeomorphism). Associated with $\tau$ there is an isomorphism $\phi$ of $\Gamma_{2}$ onto $\Gamma_{1}$, defined by

$$
(x, \phi(\gamma))=\left(\tau^{-1}(x), \gamma\right) \quad\left(x \in G_{1}, \gamma \in \Gamma_{2}\right),
$$

and there is a positive constant $k$ such that

$$
m_{1}(\tau E)=k \cdot m_{2}(E)
$$

where $m_{i}$ denotes the Haar measure of $G_{i}$ (this depends on the uniqueness theorem for Haar measure).

Fix some $\gamma_{0} \in \Gamma_{2}$, and let $T$ be the mapping of $M\left(G_{1}\right)$ into $M\left(G_{2}\right)$ defined by

$$
(T \mu)(E)=\int_{\tau E}\left(\tau^{-1}(y), \gamma_{0}\right) d \mu(y) \quad\left(\mu \in M\left(G_{1}\right)\right) .
$$

If we substitute an absolutely continuous $\mu$ into (3.1.3), so that $d \mu(x)=f(x) d m_{1}(x)$ for some $f \in L^{1}\left(G_{1}\right)$, a simple computation shows that $T$ carries $L^{1}\left(G_{1}\right)$ into $L^{1}\left(G_{2}\right)$, and that

$$
(T f)(x)=k \cdot\left(x, \gamma_{0}\right) \cdot f(\tau(x)) \quad\left(x \in G_{2}, f \in L^{1}\left(G_{1}\right)\right) .
$$

The Fourier transforms of (3.1.3) and (3.1.4) are easily seen to be

$$
\text { (3.1.5) }\left\{\begin{array}{l}
(T \mu) \uparrow(\gamma)=\hat{\mu}\left(\phi(\gamma)-\phi\left(\gamma_{0}\right)\right), \\
(T f) \frown(\gamma)=f\left(\phi(\gamma)-\phi\left(\gamma_{0}\right)\right)
\end{array} \quad\left(\mu \in M\left(G_{1}\right), f \in L^{1}\left(G_{1}\right), \gamma \in \Gamma_{2}\right) .\right.
$$

Since $\phi$ is an isomorphism onto $\Gamma_{1},(3.1 .5)$ shows that $T$ is an isomorphism of $M\left(G_{1}\right)$ onto $M\left(G_{2}\right)$ whose restriction to $L\left(G_{1}\right)$ is an isomorphism onto $L^{1}\left(G_{2}\right)$; this follows from the obvious linearity of $T$, combined with the identity 


$$
(T \mu * T \lambda)=T(\mu * \lambda)
$$

$\left(\mu, \lambda \in M\left(G_{1}\right)\right)$,

which is an immediate consequence of (3.1.5). Moreover, $T$ is an isometry:

$$
\|T \mu\|=\|\mu\| \quad\left(\mu \in M\left(G_{1}\right)\right) .
$$

This is a consequence of (3.1.3).

Conversely, if $\phi$ is any isomorphism of $\Gamma_{2}$ onto $\Gamma_{1}$, then (3.1.5) induces an isomorphism $T$ between the algebras $M\left(G_{i}\right)$ and also between the algebras $L^{1}\left(G_{i}\right) ; \phi$ determines $\tau$ via (3.1.1), and $T$ satisfies (3.1.3), (3.1.4), and (3.1.7).

The following questions now arise quite naturally: Are there any other isomorphisms between these group algebras? Can nonisomorphic groups have isomorphic group algebras? If the answer is affirmative (a simple example of this sort, in which the groups are finite, was given by Wendel [32]), to what extent does the structure of $L^{1}(G)$ or of $M(G)$ determine the structure of $G$ ?

3.2. Suppose, then, that $T$ is an isomorphism of $L^{1}\left(G_{1}\right)$ onto $L^{1}\left(G_{2}\right)$. Since $\Gamma_{i}$ is the maximal ideal space of $L^{1}\left(G_{i}\right), T$ induces a homeomorphism $\alpha$ of $\Gamma_{2}$ onto $\Gamma_{1}$, such that

$$
(T f) \wedge(\gamma)=\hat{f}(\alpha(\gamma)) \quad\left(f \in L^{1}\left(G_{1}\right), \gamma \in \Gamma_{2}\right) .
$$

Helson has shown that $T$ can be extended to an isomorphism of $M\left(G_{1}\right)$ onto $M\left(G_{2}\right)$ [8]; his proof depends on the following characterization of the F. S. transforms: a function $g$ on $\Gamma_{i}$ is a F.S. transform if and only if the mapping $\hat{f} \rightarrow g \cdot \hat{f}$ carries every Fourier transform to a Fourier transform. The action of $T$ on $M\left(G_{1}\right)$ is described by

$$
(T \mu) \uparrow(\gamma)=\hat{\mu}(\alpha(\gamma)) \quad\left(\mu \in M\left(G_{1}\right), \gamma \in \Gamma_{2}\right) .
$$

Put $\mu_{x}=T e_{x}$, where, for every $x \in G_{1}, e_{x}$ is the measure of mass 1 concentrated at the point $x$. Since $e_{x} * e_{y}=e_{x+y}$, we also have

$$
\mu_{x+y}=\mu_{x} * \mu_{y} \quad\left(x, y \in G_{1}\right),
$$

and since $T$ is an isomorphism between two semi-simple Banach algebras, $T$ is bounded [21, p. 76]. We conclude that $T$ induces a bounded homomorphism $x \rightarrow \mu_{x}$ of $G_{1}$ into $M\left(G_{2}\right)$.

In terms of $\alpha$, the conclusion is (by (3.2.2))

$$
\hat{\mu}_{x}(\gamma)=(-x, \alpha(\gamma))
$$$$
\left(x \in G_{1}, \gamma \in \Gamma_{2}\right) .
$$

3.3. The following information has so far been obtained by determining all bounded homomorphisms $x \rightarrow \mu_{x}$ or, equivalently, by investigating for which homeomorphisms $\alpha$ the functions $(-x, \alpha(\gamma))$ are F. S. transforms of uniformly bounded measures $\mu_{x}$ : 
(a) If $\Gamma_{1}$ (hence also $\Gamma_{2}$ ) is connected, then $\alpha$ is an isomorphism of $\Gamma_{2}$ onto $\Gamma_{1}$, followed by a translation of $\Gamma_{1}[1]$.

The simple isomorphisms discussed in 3.1 are thus the only ones in this situation, and we can state a corollary:

If $L\left(G_{1}\right)$ and $L\left(G_{2}\right)$ are isomorphic, and if $\Gamma_{1}$ is connected, then $G_{1}$ and $G_{2}$ are isomorphic.

The crucial step in the proof of (a) is the analysis of (3.2.4) if $\Gamma_{2}$ is the real line [1]:

(b) Let $f$ be a function on the real line, and suppose there are measures $\mu_{n}$ on the line, with $\left\{\left\|\mu_{n}\right\|\right\}$ bounded and $\hat{\mu}_{n}=f^{n}$, for $n=0, \pm 1, \pm 2, \cdots$. Then there exist real numbers $a$ and $b$ such that

$$
f(t)=e^{i(a t+b)} \quad(-\infty<t<\infty) .
$$

Note that the mapping $t \rightarrow a t+b$ (with $a \neq 0$ ) is an automorphism of the line, followed by a translation.

(c) If $\|T f\| \leqq\|f\|$ for all $f \in L^{1}\left(G_{1}\right)$, then $T$ is of the form (3.1.4).

This is proved in [8]; Wendel [33] has obtained this result even for noncommutative locally compact groups.

CoRollary. If there is an isometric isomorphism between $L^{1}\left(G_{1}\right)$ and $L^{1}\left(G_{2}\right)$, then $G_{1}$ and $G_{2}$ are isomorphic.

(d) An analysis of (3.2.3) in the case $G_{1}=G_{2}=C$ (the circle group) has led to the following result $[23]$ ( $J$ again denotes the integer group):

$A$ one-to-one mapping $\alpha$ of $J$ onto $J$ induces an automorphism of $L^{1}(C)$ (and of $M(C)$ ) if and only if there is a mapping $\beta$ of $J$ onto $J$ which differs from $\alpha$ in only a finite number of places, such that

$$
\beta(n+g)+\beta(n-g)=2 \beta(n)
$$

for some $g>0$ and all $n \in J$.

In other words, the series $\sum_{-\infty}^{\infty} c(\alpha(n)) e^{i n \theta}$ is a Fourier series whenever $\sum_{-\infty}^{\infty} c(n) e^{i n \theta}$ is a Fourier series, if and only if $\alpha$ is of the above form (and similarly for F. S. series). The endomorphisms of $L^{1}(C)$ are also determined in [23].

The mappings $\beta$.which satisfy (3.3.2) can be described in a different way: suppose

$$
J=A_{1} \cup \cdots \cup A_{k}=B_{1} \cup \cdots \cup B_{k},
$$

where each $A_{i}$ and each $B_{i}$ is an infinite arithmetic progression, and $A_{i} \cap A_{j}=B_{i} \cap B_{j}=0$ if $i \neq j$. Let $\beta$ map $A_{i}$ linearly onto $B_{i}$ in any way whatsoever (the action of $\beta$ on $A_{i}$ is independent of its action on $A_{j}$ ). Then $\beta$ satisfies (3.3.2), and every $\beta$ satisfying (3.3.2) is obtained in this way. 
(e) A slight modification of the work in [23] establishes the following [26]:

$L^{1}(G)$ and $L^{1}(C)$ are isomorphic if and only if $G$ is a direct sum $C+F$, where $F$ is a finite abelian group.

If $G=C+F$, the isomorphisms of $L^{1}(G)$ onto $L^{1}(C)$ are again induced by mappings $\alpha$ of $J$ onto $\Gamma$ which are of the form described in $(\mathrm{d})$.

3.4. Let $T$ now be an isomorphism of $M\left(G_{1}\right)$ onto $M\left(G_{2}\right)$; since $\Delta_{i}$ is the maximal ideal space of $M\left(G_{i}\right), T$ induces a homeomorphism $\alpha$ of $\Delta_{2}$ onto $\Delta_{1}$, such that

$$
(T \mu)-(\gamma)=\hat{\mu}(\alpha(\gamma)) \quad\left(\mu \in M\left(G_{1}\right), \gamma \in \Delta_{2}\right) .
$$

We saw in 3.2 that every isomorphism of $L^{1}\left(G_{1}\right)$ onto $L^{1}\left(G_{2}\right)$ extends to $M\left(G_{1}\right)$. Is it true, conversely, that the restriction of $T$ to $L^{1}\left(G_{1}\right)$ maps $L^{1}\left(G_{1}\right)$ onto $L^{1}\left(G_{2}\right)$ ? (Or, is it true that every automorphism of $M(G)$ preserves $L^{1}(G)$ ?) For this it is clearly necessary that the $\alpha$ defined by (3.4.1) should map $\Gamma_{2}$ onto $\Gamma_{1}$. But this necessary condition is also sufficient:

Let $M_{c}\left(G_{i}\right)$ be the set of all $\mu \in M\left(G_{i}\right)$ such that $\hat{\mu}$ vanishes outside some compact subset of $\Gamma_{i}$. If $\mu \in M_{c}\left(G_{i}\right)$, then $\hat{\mu} \in L^{2}\left(\Gamma_{i}\right)$. It follows that $M_{c}\left(G_{i}\right) \subset L^{1}\left(G_{i}\right)$; moreover, $M_{c}\left(G_{i}\right)$ is dense in $L^{1}\left(G_{i}\right)$. Thus $T$ maps $M_{c}\left(G_{1}\right)$ onto $M_{c}\left(G_{2}\right)$ if $\alpha\left(\Gamma_{2}\right)=\Gamma_{1}$, and this implies that $T$ maps $L^{1}\left(G_{1}\right)$ onto $L^{1}\left(G_{2}\right)$.

We next observe that if $\alpha$ is given by (3.4.1), then $\alpha\left(\bar{\Gamma}_{2}\right)=\bar{\Gamma}_{1} .(\bar{\Gamma}$ denotes the closure of $\Gamma$ in $\Delta$.) This follows from the fact that $\bar{\Gamma}_{i}$ is the unique closed subset $E$ of $\Delta_{i}$ which is minimal with respect to the following property: if $\sigma \in M\left(G_{i}\right)$ and if $\hat{\sigma}=0$ at every point of $E$, then $\sigma=0$.

If we now assume that $\Gamma_{1}$ satisfies the first axiom of countability, we can prove that $\alpha\left(\Gamma_{2}\right)=\Gamma_{1}$ (this is undoubtedly true without any countability restriction, but I have no proof that covers the general case): Since $\alpha\left(\bar{\Gamma}_{2}\right)=\bar{\Gamma}_{1}$, there is a dense open subset $V$ of $\Gamma_{2}$ such that $\alpha(V) \subset \Gamma_{1}$, and for any $\gamma_{0} \in \Gamma_{2}$ there is a simple countable sequence $\left\{\gamma_{n}\right\}$, with $\gamma_{n} \in V$, such that $\gamma_{n} \rightarrow \gamma_{0}$. By (3.4.1),

$$
\hat{\mu}\left(\alpha\left(\gamma_{n}\right)\right) \rightarrow \hat{\mu}\left(\alpha\left(\gamma_{0}\right)\right)
$$$$
\left(\mu \in M\left(G_{1}\right)\right) .
$$

In particular, if $\mu=e_{x}$ (see 3.2), then

$$
\left(-x, \alpha\left(\gamma_{n}\right)\right) \rightarrow\left(-x, \alpha\left(\gamma_{0}\right)\right)
$$$$
\left(x \in G_{1}\right) .
$$

If $\left\{\alpha\left(\gamma_{n}\right)\right\}$ lies in a compact subset of $\Gamma_{1}$, then $\alpha\left(\gamma_{0}\right) \in \Gamma_{1}$. If not, there is a subsequence, again denoted by $\left\{\alpha\left(\gamma_{n}\right)\right\}$, which tends to infinity in $\Gamma_{1}$. Integrating (3.4.3) over some set $A$ of positive finite 
Haar measure, and applying the Riemann-Lebesgue theorem, we obtain

$$
\int_{A}\left(-x, \alpha\left(\gamma_{n}\right)\right) d m_{1}(x) \rightarrow 0 \quad(n \rightarrow \infty) ;
$$

by the Lebesgue convergence theorem (and this is where a simple countable sequence is needed), (3.4.3) implies

$$
\int_{A}\left(-x, \alpha\left(\gamma_{0}\right)\right) d m_{1}(x)=0 .
$$

Since $A$ was arbitrary, $\left(-x, \alpha\left(\gamma_{0}\right)\right)=0$ for almost all $x$, contradicting (3.4.3).

Thus $\alpha\left(\gamma_{0}\right) \in \Gamma_{1}$. It follows that $\alpha\left(\Gamma_{2}\right) \subset \Gamma_{1}$. Since the same argument applies to $\alpha^{-1}$, the following theorem is proved:

3.5. THEOREM. If $T$ is an isomorphism of $M\left(G_{1}\right)$ onto $M\left(G_{2}\right)$ and if $\Gamma_{1}$ satisfies the first axiom of countability, then $T$ maps $L^{1}\left(G_{1}\right)$ onto $L^{1}\left(G_{2}\right)$.

The structure theorem for locally compact abelian groups [ 31 , p. 110] shows that the countability assumption imposed on $\Gamma_{1}$ is equivalent to the following condition on $G_{1}$ : There is an open-closed subgroup $G_{0}$ of $G_{1}$ which is the direct sum of a Euclidean space and a compact group, and which has only a countable number of cosets in $G_{1}$. In other words, the Haar measure of $G_{1}$ is $\sigma$-finite.

3.6. In 3.2, a condition was found (namely (3.2.4)) which is necessary for the homeomorphism $\alpha$ to induce an isomorphism of $L^{1}\left(G_{1}\right)$ onto $L^{1}\left(G_{2}\right)$, via (3.2.1). But this condition is also sufficient, as shown by the following theorem, in conjunction with $\$ 3.4$ :

THEOREM. Let $\alpha$ be a mapping (not assumed to be continuous) of $\Gamma_{2}$ into $\Gamma_{1}$, with the following property: for each $x \in G_{1}$ there is a $\mu_{x} \in M\left(G_{2}\right)$ such that

$$
\hat{\mu}_{x}(\gamma)=(-x, \alpha(\gamma)) \quad\left(x \in G_{1}, \gamma \in \Gamma_{2}\right),
$$

and $\left\|\mu_{x}\right\| \leqq A<\infty$, where $A$ is independent of $x$. Then for every $\sigma \in M\left(G_{1}\right)$, the function $f$ defined by

$$
f(\gamma)=\hat{\sigma}(\alpha(\gamma))=\int_{G_{2}}(-x, \alpha(\gamma)) d \sigma(x) \quad\left(\gamma \in \Gamma_{2}\right)
$$

is a Fourier-Stieltjes transform on $\Gamma_{2}$.

Proof. Choose $\gamma_{1}, \cdots, \gamma_{p} \in \Gamma_{2}$ and choose complex numbers $a_{1}, \cdots, a_{p}$. Then 


$$
\begin{aligned}
\sum_{n} a_{n} f\left(\gamma_{n}\right) & =\sum_{n} a_{n} \hat{\sigma}\left(\alpha\left(\gamma_{n}\right)\right) \\
& =\int_{G_{2}} \sum_{n} a_{n} \hat{\mu}_{x}\left(\gamma_{n}\right) d \sigma(x) \\
& =\int_{G_{2}}\left\{\int_{G_{2}} \sum_{n} a_{n}\left(-y, \gamma_{n}\right) d \mu_{x}(y)\right\} d \sigma(x),
\end{aligned}
$$

so that

$$
\left|\sum_{n} a_{n} f\left(\gamma_{n}\right)\right| \leqq\|\sigma\| \cdot A \cdot \sup \left|\sum_{n} a_{n}\left(y, \gamma_{n}\right)\right| \quad\left(y \in G_{2}\right) .
$$

Since this holds for arbitrary $\gamma_{1}, \cdots, \gamma_{p}$ and $a_{1}, \cdots, a_{p}, f$ is a F. S. transform, by a theorem of Eberlein [4], whose original form is due to Bochner [2].

IV. Idempotent measures. 4.1. The discussion of isomorphisms of group algebras has led us to the problem of determining the bounded homomorphisms of $G_{1}$ into $M\left(G_{2}\right)$, given by

$$
\mu_{x+y}=\mu_{x} * \mu_{y}
$$$$
\left(x, y \in G_{1}\right) \text {. }
$$

A special case of this problem deserves attention: if $x=y=0,(4.1 .1)$ becomes

$$
\mu=\mu^{2} \text {. }
$$

That is to say, we are led to the problem of finding all idempotents in $M(G)$; clearly, we cannot hope to solve (4.1.1) unless we can solve (4.1.2).

(a) This problem has two other aspects which make it interesting: There is a one-to-one correspondence between the set of all bounded projections $P$ of $L^{1}(G)$ which commute with translations, and the set of all idempotent $\mu \in M(G)$, given by

$$
P f=f * \mu .
$$

(b) One of the basic problems of Fourier analysis is that of determining just which functions on $\Gamma$ are $F$. S. transforms. If we know all idempotents in $M(G)$, then we know all simple functions (i.e., those with only a finite set of values) which are F. S. transforms on $\Gamma$.

4.2. If $\mu \in M(G)$ and $\mu=\mu^{2}$, then $\hat{\mu}=\hat{\mu}^{2}$, so that $\hat{\mu}(\gamma)=0$ or 1 for all $\gamma \in \Gamma$. Let $S(\mu)$ be the set of all $\gamma \in \Gamma$ at which $\hat{\mu}(\gamma)=1$. The problem of finding all idempotents in $M(G)$ is thus equivalent to that of find- 
ing all subsets of $\Gamma$ whose characteristic functions are F. S. transforms.

The continuity of $\hat{\mu}$ implies that $S(\mu)$ must be open-closed. A particular case of the problem can therefore be solved immediately: if $\Gamma$ is connected, then $M(G)$ has no nontrivial idempotents (there are always two trivial ones: $\mu=0$ and $\mu=u$, the unit of $M(G))$.

It is interesting that the problem reduces to the consideration of compact $G$ :

4.3. THEOREM [27]. If $\mu \in M(G)$ and if $\mu=\mu^{2}$, then $\mu$ is concentrated on a compact subgroup $K$ of $G$ (and hence $\mu$ may be regarded as an element of $M(K))$.

4.4. We may thus suppose that $G$ is compact. If $\Lambda$ is a subgroup of $\Gamma$ and $H$ is the subgroup of $G$ which consists of all $x$ such that $(x, \gamma)=1$ for all $\gamma \in \Lambda$, then $m_{H}$ (the Haar measure of $H$ ) is an idempotent element of $M(G)$, and $\Lambda=S\left(m_{H}\right)$. If we define $\mu$ by

$$
d \mu(x)=(x, \gamma) d m_{H}(x)
$$

for some $\gamma \in \Gamma$, then $\mu=\mu^{2}$, and $S(\mu)=\Lambda+\gamma$. Thus every coset of $\Lambda$ is $S(\mu)$ for some idempotent $\mu$.

Let $R(\Gamma)$ be the smallest family of subsets of $\Gamma$ which contains all cosets of all subgroups of $\Gamma$, and which is closed under complementation and finite intersection. We call $R(\Gamma)$ the coset ring of $\Gamma$.

Since $S(\mu) \cap S(\lambda)=S(\mu * \lambda)$ and $S(u-\mu)$ is the complement of $S(\mu)$, the above remark implies that every set in $R(\Gamma)$ is $S(\mu)$ for some idempotent $\mu \in M(G)$. The converse is probably true too:

4.5. Conjecture. $A$ set $E \subset \Gamma$ is $S(\mu)$ for some idempotent $\mu \in M(G)$ if and only if $E$ belongs to $R(\Gamma)$.

4.6. If $G=C$, this conjecture is true, as was proved by Helson $[9 ; 10]$ (his result preceded and suggested the conjecture) and I have recently extended it to all finite-dimensional torus groups [27], using the decomposition into irreducible measures described in 4.7 , as well as some work on multiple Fourier series which was done by Helson and Lowdenslager [12].

4.7. I call an idempotent $\mu \in M(G)$ irreducible if the smallest compact subgroup $H$ of $G$ on which $\mu$ is concentrated has the following property: if $K$ is a compact subgroup of $H$ and if $m(K)=0$, where $m$ is the Haar measure of $H$, then $\mu(E)=0$ for all subsets $E$ of $K$.

The above-mentioned decomposition theorem states: if $\mu \in M(G)$ and $\mu=\mu^{2}$, then there are integers $a_{1}, \cdots, a_{n}$ and irreducible idempotents $\mu_{1}, \cdots, \mu_{n}$ in $M(G)$, such that $\mu=a_{1} \mu_{1}+\cdots+a_{n} \mu_{n}$.

In order to prove the conjecture in its full generality, one has there- 
fore to show that every irreducible idempotent measure $\mu$ is absolutely continuous with respect to the Haar measure of the smallest compact group on which $\mu$ is concentrated.

4.8. One other special case should be mentioned: If $\mu \in M(G)$, $\mu=\mu^{2}$, and $\|\mu\|=1$, then it is easily seen that $S(\mu)$ is a coset of a subgroup of $\Gamma$. In particular, if $\mu=\mu^{2}$ and $\mu \geqq 0$, then $\mu$ is the Haar measure of a compact subgroup of $G$; this last statement is true even on noncommutative compact groups [34].

V. Transformations of Fourier-Stieltjes transforms. 5.1. If $\mathcal{F}$ is a family of functions, defined on some set $S$, there are two kinds of transformations of $\mathcal{F}$ that are often of interest: the domain transformations and the range transformations of $\mathfrak{F}$.

A domain transformation of $\mathcal{F}$ is a mapping $\alpha$ of $S$ into $S$, such that $f \circ \alpha \in \mathcal{F}$ whenever $f \in \mathcal{F}$; here $f \circ \alpha$ is the function defined by $(f \circ \alpha)(x)$ $=f(\alpha(x))$, for $x \in S$. In Chapter III, domain transformations of Fourier transforms and of F. S. transforms were discussed. If $\mathcal{F}$ is a linear space, domain transformations of $\mathfrak{F}$ are linear operations on $\mathfrak{F}$.

A function $\Phi$, defined on a set $E$ in the complex plane, may be called a range transformation of $\mathcal{F}$ if $\Phi \circ f \in \mathcal{F}$ for every $f \in \mathcal{F}$ whose range lies in $E$. For brevity, we shall use the phrase " $\Phi$ operates in $F$ " to describe this situation. For example, if $\mathcal{F}$ is an algebra (with pointwise multiplication) and if $\Phi(z)$ is a polynomial in $z$, then $\Phi$ operates in $\mathcal{F}$ (no matter what $E$ is).

We shall be concerned with the problem of determining those $\Phi$ which operate in algebras of Fourier transforms and of F.S. transforms.

5.2. Definition of $A, F$, And $F S$. We let $A$ denote the set of all functions on $C$ which are sums of absolutely convergent trigonometric series; $F$ is the set of all sequences of Fourier coefficients (i.e., $F$ is the set of Fourier transforms of the functions in $\left.L^{1}(C)\right)$; and $F S$ is the set of all sequences of F. S. coefficients (i.e., the set of F. S. transforms of measures in $M(C)$ ).

5.3. The earliest theorem in this circle of ideas is due to Wiener and Lévy $[35 ; 20]:$ If $f \in A$ and if $\Phi$ is analytic on the range of $f$, then $\Phi \circ f \in A$.

It is natural to ask whether this sufficient condition of analyticity can be weakened, but the search for strong necessary conditions has been successful only quite recently.

Let us assume that $\Phi$ is defined on the closed interval $I=[-1,1]$. In 1954 , Kahane $[14 ; 15]$ proved that $\Phi$ does not operate in $A$ if $\Phi(x)=|x|$, and more or less simultaneously I proved the same for $\Phi(x)=(1+x)^{1 / 2}$, and for some similar functions [22]. In 1956, I 
proved that if $\Phi$ operates in $F$, then $\Phi$ must satisfy a Lipschitz condition of order 1 in some neighborhood of the origin; if $\Phi$ operates in $F S$, then the Lipschitz condition holds uniformly on $I$ [24]. Kahane [16] countered by proving that this necessary condition was not sufficient: he showed that $\Phi$ does not operate in $F$ if $\Phi(x)=|x|$.

Last spring a bigger step was made: Kahane [17] constructed infinitely differentiable functions which do not operate in $A$. But the final results were only obtained within the last month:

5.4. THEOREM. Suppose $\Phi$ is a complex function defined on the interval $I=[-1,1]$. [19].

(a) $\Phi$ operates in $A$ if and only if $\Phi$ is analytic at every point of $I$

(b) $\Phi$ operates in $F$ if and only if $\Phi$ is analytic in some neighborhood of the origin [11].

(c) $\Phi$ operates in FS if and only if $\Phi$ can be extended to an entire function in the complex plane [18].

5.5. If $\Phi$ is defined in the whole plane (or in a region), analogous conclusions are obtained, with "real-analytic" in place of "analytic." But 5.4(c) also has the following corollary [18].

Suppose $\Phi$ is defined in the whole complex plane. Then $\Phi$ operates in the algebra of the Gelfand transforms of the measures in $M(C)$ if and only if $\Phi$ is an entire function of a complex variable.

This is a much stronger result than Theorem 2.4(a), where the function $\Phi(z)=\bar{z}$ is ruled out. However, it should be recorded that Theorem 2.4(a) led to the conjecture that 5.4(c) might be true.

5.6. I would like to end with a brief sketch of the proof of Theorem 5.4(c). For $\mu \in M(C)$, let $\Phi \circ \mu$ denote the measure $\sigma$ such that $\hat{\sigma}(n)=\Phi(\hat{\mu}(n))$ for $n=0, \pm 1, \pm 2, \cdots$. One first proves that to each $r>0$ there is an $M(r)<\infty$ such that $\|\Phi \circ \mu\| \leqq M(r)$ whenever $\|\mu\| \leqq r$ and $\hat{\mu}(n) \in I$, and one defines

$$
\Psi(x)=\Phi(R \sin x)=\sum_{-\infty}^{\infty} c_{n} e^{i n x} \quad(-\infty<x<\infty)
$$

for some $R$ satisfying $0<R<1$. Then for every real number $a$,

$$
\|\Psi \circ(\mu+a)\| \leqq M\left(R e^{r}\right)=M
$$

whenever $\|\mu\| \leqq r$ and $\hat{\mu}$ is real on $J$. Since, formally,

$$
\Psi \circ(\mu+a)=\sum_{-\infty}^{\infty} c_{n} e^{i n \mu} e^{i n a},
$$

it is reasonable to expect that 


$$
\begin{aligned}
\left\|c_{n} e^{i n \mu}\right\|=\left\|\frac{1}{2 \pi} \int_{-\pi}^{\pi} \Psi \circ(\mu+a) e^{-i n a} d a\right\| & \leqq M \\
(n & =0, \pm 1, \pm 2, \cdots),
\end{aligned}
$$

if $\|\mu\| \leqq r$ and $\hat{\mu}$ is real on $J$. This is not hard to prove, and if we take $\mu$ as in Lemma 2.3 , we can conlude that

$$
\left|c_{n}\right| e^{|n| r} \leqq M \quad(n=0, \pm 1, \pm 2, \cdots) .
$$

Consequently the Fourier series in (5.6.1) converges to an analytic function in the strip $|y|<r$. This is true for all $r$, so that $\Psi$ is an entire function.

Let us do this for two different values of $R$, say $R_{1}$ and $R_{2}$, and call the corresponding entire functions $\Psi_{1}$ and $\Psi_{2}$. We then have, in some neighborhood of the origin,

$$
\Phi(x)=\Psi_{1}\left(\arcsin \frac{x}{R_{1}}\right)=\Psi_{2}\left(\arcsin \frac{x}{R_{2}}\right) .
$$

The first representation of $\Phi$ shows that $R_{1},-R_{1}$, and $\infty$ are the only possible singular points of $\Phi$; the second representation shows that $R_{2},-R_{2}$, and $\infty$ are the only possibilities. Since $R_{1} \neq R_{2}, \Phi$ can be extended to an entire function.

Postscript (added February 4, 1959). Since this address was delivered, the following new developments have occurred in the subject under consideration:

(1) P. J. Cohen has characterized all idempotents in $M(G)$; the conjecture 4.5 is correct.

(2) Using this result, he has also characterized all homomorphisms of $L^{1}\left(G_{1}\right)$ into $M\left(G_{2}\right)$. A summary of this work will appear shortly as a Research Announcement in this Bulletin.

(3) One can deduce from Cohen's work that Theorem 3.5 is true without any countability restriction on $\Gamma_{1}$.

(4) Theorem 5.4 has been extended to locally compact abelian groups in general. Three cases have to be distinguished (we assume $\Phi(0)=0, \Phi$ is defined on $I$, and we let $A(\Gamma), B(\Gamma)$ denote the algebras of all Fourier transforms and F. S. transforms, respectively, on $\Gamma$ ):

(a) If $\Gamma$ is not discrete, $\Phi$ operates in $A(\Gamma)$ if and only if $\Phi$ is analytic on $I$.

(b) If $\Gamma$ is discrete, $\Phi$ operates in $A(\Gamma)$ if and only if $\Phi$ is analytic in some neighborhood of the origin.

(c) If $\Gamma$ is not compact, $\Phi$ operates in $B(\Gamma)$ if and only if $\Phi$ can be extended to an entire function in the complex plane. 
Proofs of these results are being published jointly by the authors of $[11 ; 18 ; 19]$.

\section{BIBLIOGRAPHY}

1. Arne Beurling and Henry Helson, Fourier-Stieltjes transforms with bounded powers, Math. Scand. vol. 1 (1953) pp. 120-126.

2. Salomon Bochner, $A$ theorem on Fourier-Stieltjes integrals, Bull. Amer. Math. Soc. vol. 40 (1943) pp. 271-276.

3. Henri Cartan and Roger Godement, Thêorie de la dualité et analyse harmonique dans les groupes abéliens localement compacts, Ann. Sci. Ecole Norm. Sup. (3) vol. 64 (1947) pp. 79-99.

4. W. F. Eberlein, Characterizations of Fourier-Stieltjes transforms, Duke Math. J. vol. 22 (1955) pp. 465-468.

5. I. M. Gelfand, D. A. Raikov and G. E. Silov, Commutative normed rings, Amer. Math. Soc. Translations Ser. 2, vol. 5 pp. 115-220 [Uspehi Mat. Nauk (N. S.) 1, no. 2 vol. 12 (1946) pp. 48-146].

6. Alfred Haar, Der Massbegriff in der Theorie der kontinuierlichen Gruppen, Ann. of Math. vol. 34 (1933) pp. 147-169.

7. Paul Halmos, Measure theory, New York, 1950.

8. Henry Helson, Isomorphisms of abelian group algebras, Ark. Mat. vol. 2 (1953) pp. $475-487$.

9. - Note on harmonic functions, Proc. Amer. Math. Soc. vol. 4 (1953) pp. $686-691$. 242.

10. - On a theorem of Szegö, Proc. Amer. Math. Soc. vol. 6 (1955) pp. 235-

11. Henry Helson and J. P. Kahane, Sur les fonctions opérant dans les algèbres de transformées de Fourier de suites ou de fonctions sommables, C. R. Acad. Sci. Paris vol. 247 (1958) pp. 626-628.

12. Henry Helson and David Lowdenslager, Prediction theory and Fourier series in several variables, Acta Math. vol. 99 (1958) pp. 165-202.

13. Edwin Hewitt, The asymmetry of certain algebras of Fourier-Stieltjes transforms, Michigan Math. J. vol. 5 (1958) pp. 149-158.

14. J. P. Kahane, Sur les fonctions sommes de séries trigonométriques absolument convergentes, C. R. Acad. Sci. Paris vol. 240 (1955) pp. 36-37.

15. - - Sur certaines classes de séries de Fourier absolument convergentes, J. Math. Pures Appl. vol. 35 (1956) pp. 249-259. 271.

16. ——, Sur un problème de Littlewood, Indag. Math. vol. 19 (1957) pp. 268-

17. - Sur un thêorème de Wiener-Lévy, C. R. Acad. Sci. Paris vol. 246 (1958) pp. 1949-1951.

18. J. P. Kahane and Walter Rudin, Caractérisation des fonctions qui opérent sur les coefficients de Fourier-Stieltjes, C. R. Acad. Sci. Paris vol. 247 (1958) pp. 773-775.

19. Yitzhak Katznelson, Sur les fonctions opérant sur l'algèbre des séries de Fourier absolument convergentes, C. R. Acad. Sci. Paris vol. 247 (1958) pp. 404-406.

20. Paul Lévy, Sur la convergence absolue des sêries de Fourier, Compositio Math. vol. 1 (1934) pp. 1-14.

21. L. H. Loomis, An introduction to abstract harmonic analysis, New York, 1953.

22. Walter Rudin, Nonanalytic functions of absolutely convergent Fourier series, Proc. Nat. Acad. Sci. U.S.A. vol. 41 (1955) pp. 238-240. 
23. - The automorphisms and the endomorphisms of the group algebra of the unit circle, Acta Math. vol. 95 (1956) pp. 39-55.

24. - Transformations des coefficients de Fourier, C. R. Acad. Sci. Paris vol. 243 (1956) pp. 638-640.

25. - Independent perfect sets in groups, Michigan Math. J. vol. 5 (1958) pp. $159-161$.

26. - On isomorphisms of group algebras, Bull. Amer. Math. Soc. vol. 64 (1958) pp. 167-169.

27. - Idempotent measures on abelian groups, to appear in Pacific J. Math.

28. Raphael Salem, On sets of multiplicity for trigonometrical series, Amer. J. Math. vol. 64 (1942) pp. 531-538.

29. G. E. Silov, On decomposition of a commutative normed ring in a direct sum of ideals, Mat. Sb. N. S. vol. 32 (74) (1953) pp. 353-364.

30. $\mathrm{Yu}$. A. Sreider, The structure of maximal ideals in rings of measures with convolution, Amer. Math. Soc. Translation, no. 81, Providence, 1953.

31. André Weil, L'intégration dans les groupes topologiques, Paris, 1953.

32. J. G. Wendel, On isometric isomorphism of group algebras, Pacific J. Math. vol. 1 (1951) pp. 305-311.

33. - Left centralizers and isomorphisms of group algebras, Pacific J. Math. vol. 2 (1952) pp. 251-261.

34. - Haar measure and the semigroup of measures on a compact group, Proc. Amer. Math. Soc. vol. 5 (1954) pp. 923-926.

35. Norbert Wiener, Tauberian theorems, Ann. of Math. vol. 33 (1932) pp. 1-100.

36. Norbert Wiener and R. H. Pitt, On absolutely convergent Fourier-Stieltjes transforms, Duke Math. J. vol. 4 (1938) pp. 420-436.

37. J. H. Williamson, On constructions of Wiener-Pitt and Sreider, Communication at International Congress of Mathematicians, Edinburgh, 1958.

UNIVERSITY OF ROCHESTER and YALE UNIVERSITY 